\section{How flies time}

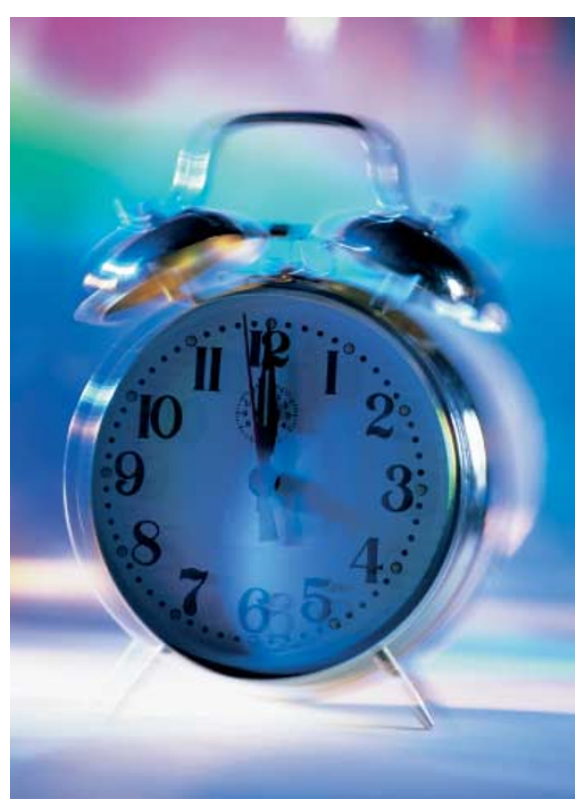

Two papers published in Nature use different techniques to show that separate groups of circadian neurons control the characteristic morning and evening bouts of activity in the fruitfly Drosophila melanogaster.

When flies are kept in a cycle consisting of $12 \mathrm{~h}$ of light and $12 \mathrm{~h}$ of darkness, their activity peaks twice a day, around the times that the lights are switched on (morning) or off (evening). They become more active before the change in lighting, showing that their internal circadian clock allows them to anticipate the change in illumination.

Circadian rhythmicity in D. melanogaster depends on about 100 'clock' neurons, which are found in six clusters in the brain. Stoleru et al. and Grima et al. used targeted gene expression techniques to investigate the specific contributions of different groups of cells to the morning and evening peaks in activity. Stoleru and colleagues used the cryptochrome (cry) gene driver to drive expression of the proapoptotic gene hid, to kill circadian neurons that expressed the circadian photoreceptor $c r y$, which produced a severe lack of circadian rhythmicity. The flies did not show anticipatory activity before lights-on or lights-off, and in constant darkness they were arrhythmic.

Ablation of one subset of neurons - the ventral-lateral $\mathrm{LN}_{\mathrm{v}}$ neurons, which express the neuropeptide PDF (pigment-dispersing factor) - suppresses the morning anticipatory peak of activity. When the authors generated flies in which only the rest of the cry-expressing neurons - those that did not also express PDF - were ablated, these flies had a normal activity peak in the morning, but did not show activity in anticipation of lights-off in the evening.

These results indicate that the $\mathrm{LN}_{\mathrm{v}}$ neurons are responsible for the morning activity peak, and the other cry-expressing neurons, including the dorsal-lateral $\mathrm{LN}_{\mathrm{d}}$ neurons, are responsible for the evening activity peak. Grima and colleagues came to the same conclusion, by using cell-specific drivers to rescue expression of the circadian protein Per in subsets of neurons in per-null flies. When

\title{
The two faces of dopamine
}

In mammals and other animals, dopamine receptors can be split into two groups - D1like and D2-like. Writing in Nature

Neuroscience, Chase et al. describe a genetic analysis of dopamine signalling in the worm Caenorhabiditis elegans that sheds light on the antagonistic properties of these two types of receptor.

There is strong evidence from mammalian studies that signalling through D1- and D2like dopamine receptors has opposing effects on locomotor behaviour, and that the two types of receptor can signal through different neuronal $G$ proteins. To delve deeper into the mechanisms of dopaminergic signalling, the authors turned to the genetic tools that are available for C. elegans.

In a search of the C. elegans genome, using the known D1-like receptor DOP-1 and D2-like receptor DOP-2 for comparison, the authors identified a new D2-like dopamine receptor, DOP-3. Worms in which the dop-3 gene was deleted showed a reduced 'basal slowing response' - the slowing of locomotion that is normally caused when a worm encounters a bacterial lawn, and which is known to be controlled by dopamine signalling. By contrast, $d o p-1$ or dop- 2 mutants showed a normal basal slowing response. dop-3 mutants were also resistant to the paralytic effects of high concentrations of exogenous dopamine, whereas dop-1 or dop-2 mutants showed no such resistance. However, double mutants for both dop-1 and dop-3 showed a near-normal response in both cases, indicating that DOP-1 signalling antagonizes DOP-3.

To investigate the cellular basis for these effects, the authors looked at the expression of the dopamine receptors in C. elegans. Although the expression patterns of DOP-1 and DOP-3 did not initially seem to overlap, closer inspection revealed that the cholinergic motor neurons of the ventral cord expressed dop-1 strongly and dop-3 more weakly. Experiments in which expression of $d o p-1$ or $d o p-3$ was restored specifically in these motor neurons in mutant worms showed that the effects of the two receptors on locomotion were mediated by these neurons, showing that the antagonistic effects of D1-like and D2-like receptors can occur in the same cell.
What signalling pathways are responsible for these effects? In a screen for other dopamineresistant mutants, Chase et al. found nine mutations in four genes, each of which encodes a neuronal G-protein signalling molecule. These signalling molecules were components of two opposing signalling pathways that have been characterized in C. elegans and that are conserved in mammals. Mutations that either increased signalling

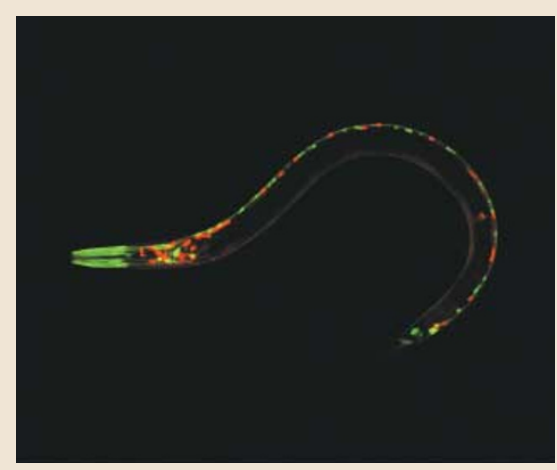

Fluorescence imaging of a worm carrying integrated transgenes that express green fluorescent protein from the dop-1 promoter and red fluorescent protein from the dop-3 promoter. Image courtesy of D. Chase, Yale University, USA. 
Per expression was rescued in the $\mathrm{LN}_{\mathrm{v}}$ neurons, the morning activity peak was restored, but when it was rescued in both the $\mathrm{LN}_{\mathrm{v}}$ and $\mathrm{LN}_{\mathrm{d}}$ neurons, both the morning and evening activity peaks were normal.

Both groups also found that the 'morning' oscillator, maintained by the $\mathrm{LN}_{\mathrm{v}}$ neurons, can be maintained in sustained darkness, whereas the 'evening' oscillator cannot, although this is difficult to reconcile with findings that the morning peak, but not the evening peak, is progressively lost when wild-type flies are placed in darkness. It is likely that these two oscillators, although they can act independently, are functionally coupled under normal circumstances to allow them to be coordinated and to respond flexibly to challenges such as seasonal changes.

Rachel Jones

(9) References and links

ORIGINAL RESEARCH PAPERS Stoleru, D. et al. Coupled oscillators control morning and evening locomotor behaviour of Drosophila. Nature 431, 862-868 (2004) | Grima, B. et al. Morning and evening peaks of activity are controlled by different clock neurons of the Drosophila brain. Nature 431, 869-873 (2004)

FURTHeR READING Hastings, M. H. et al. A clockwork web: circadian timing in brain and periphery, in health and disease. Nature Rev. Neurosci. 4, 649-661 (2003)

through the $\mathrm{G} \alpha_{\mathrm{q}}$ pathway or decreased signalling through the $\mathrm{G} \alpha_{\mathrm{o}}$ pathway resulted in dopamine resistance. These results and others fit a model in which DOP-3 signals through $\mathrm{G} \alpha_{\mathrm{o}}$ to inhibit locomotion, and DOP-1 antagonizes this effect by signalling though $\mathrm{G} \alpha$.

Dopamine signalling in worms shows striking parallels with the dopamine system in mammals. In both types of animal, D1- and D2-like receptors are expressed at different levels in the same neurons, and can have opposing effects on behaviour. As in worms, D1 receptors in mammals can activate $\mathrm{G} \alpha_{\mathrm{q}}$ signalling, and $D 2$ receptors can activate $G \alpha_{o}$ signalling, although it has not been previously shown that these pathways mediate their antagonistic effects. Moreover, dopamine in both worms and mammals can act extrasynaptically as a neurohormone. If these parallels hold true for other details of dopamine signalling, results from studies of C. elegans could give important insights into how mammalian dopamine receptors function.

\section{Rachel Jones}

60) References and links ORIGINAL RESEARCH PAPER Chase, D. L. et al. Mechanism of extrasynaptic dopamine signaling in Caenorhabditis elegans. Nature Neurosci. 7, 1096-1103 (2004) WEB SITE

Koelle lab http://info.med.yale.edu/mbb/koelle/
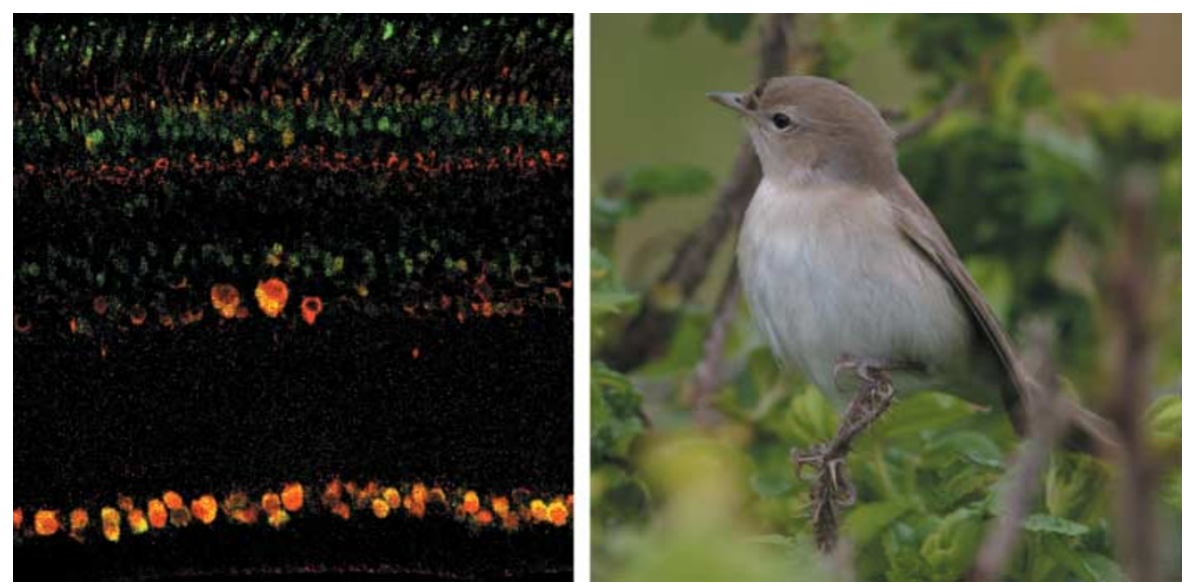

Left: retina section of garden warbler, stained with CRY1 (green) and c-Fos (red). Figure reproduced, with permission, from The National Academy of Sciences USA. Right: a garden warbler. Image courtesy of H. Mouritsen, University of Oldenburg, Germany.

\section{SENSORY SYSTEMS}

\section{Homing in on magnetic orientation}

Migratory birds use the Earth's magnetic field for homing and navigation. How they do this is not clear, but theoretical biophysicists have predicted that photoreceptor molecules in the retina might be involved in sensing magnetic fields. Reporting in the Proceedings of the National Academy of Sciences, Mouritsen and colleagues investigated the expression of cryptochromes (CRYs) - blue-green photoreceptors that are important in the regulation of circadian rhythms - in the retina and their association with neuronal activity during magnetic orientation.

Biophysicists have proposed that magnetic sensing might be based on the sensitivity of a class of chemical reactions called radical-pair reactions to magnetic fields. In a radical-pair reaction, absorption of light through a photoreceptor molecule, such as a CRY, induces the transfer of an electron from one molecule to another and results in a pair of molecules each with an unpaired electron. These unpaired electrons have particular spin states that can be affected by an external magnetic field, which might alter downstream chemical and biological events.

This theoretical analysis is supported by the fact that magnetic sensing in migratory birds is light-dependent: they can sense magnetic fields under blue and green light but become disorientated under dim red light. This observation also indicates that CRYs might be involved in radical-pair reactions in magnetoreception. In this study, Mouritsen and colleagues identified two CRY isoforms in the retina of migratory garden warblers - the cytosolic gwCRY1 and the nuclear gwCRY2. As molecules that serve as magnetoreceptors must be orientated in the cell and, therefore, are more likely to be localized in the cytosol in association with cytoskeletal proteins, the authors focused on gwCRY1.

In the retina of non-migratory zebra finches, expression of CRY1 is high during the day and drops markedly at night. In migratory garden warblers, CRY1 is expressed during both day and night in the retinal ganglion cells and a specific group of cells in the retina called displaced ganglion cells that have been implicated in magnetoreception. Interestingly, expression of CRY1 is absent in displaced ganglion cells in zebra finches.

The authors then analysed the functional relevance of CRY expression for magnetoreception by studying the expression of c-Fos and ZENK - markers of light-dependent neuronal activation - in migratory and nonmigratory birds. They found that both c-Fos and ZENK were strongly expressed in the retina of garden warblers at night during magnetic orientation, and the expression co-localized with CRY1 in all ganglion cells and displaced ganglion cells. By contrast, c-Fos is only weakly expressed during the night in the retina of zebra finches, and expression of c-Fos in displaced ganglion cells does not correlate with that of CRY1.

Although direct functional evidence is still lacking to support a definitive role for CRYs in magnetoreception, the study is an encouraging step towards unravelling the molecular mechanisms of magnetic orientation.

(2) References and links Jane Qiu ORIGINAL RESEARCH PAPER Mouritsen, $\mathrm{H}$. et al Cryptochromes and neuronal-activity markers colocalize in the retina of migratory birds during magnetic orientation. Proc. Natl Acad. Sci. USA 101, 14294-14299 (2004) FURTHER READING Ritz, T. et al. Shedding light on vertebrate magnetoreception. Neuron 34, 503-506 (2002) 